\title{
Influencia del ABP en el Nivel de Razonamiento de Estudiantes de Educación Superior
}

\section{Influence of ABP on the Reasoning Level of Higher Education Students}

\section{Leidy Lorena Herrera Briñez'}

\section{Mariela del Pilar Gómez Castro²}

Corporación Universitaria Minuto de Dios Vicerrectoría Tolima Magdalena

\section{RESUMEN}

La habilidad de razonamiento permite realizar la búsqueda de información, el análisis de la misma y dar solución a determinada situación problema por resolver; para su fortalecimiento se ha hecho necesaria la inclusión de estrategias didácticas en los procesos de enseñanza aprendizaje que

$1 \quad$ Profesor Programa de Psicología Corporación Universitaria Minuto de Dios - Vicerrectoría Tolima Magdalena Medio. Colombia. Magister en Educación. https://orcid. org/0000-0002-8528-2179. https://scholar.google.com/citat ions?authuser=1\&user=c2VsBsUAAAAJ. +573124124053 . Iherrerabri@uniminuto.edu.co

2 Profesor Programa de Psicología Corporación Universitaria Minuto de Dios - Vicerrectoría Tolima Magdalena Medio. Colombia. Magister en Educación. https://orcid.org/0000-0001-8283-8277. https://scholar. google.es/citations? user=jHI4SPwAAAAJ\&hl=es\&authus er=1.+573114526993. mgomezcast5@uniminuto.edu.co faciliten su desarrollo, una de esas estrategias es el aprendizaje basado en problemas. La presente investigación propuso como objetivo determinar la influencia de la estrategia Aprendizaje Basado en Problemas en el nivel de razonamiento de un grupo de estudiantes de educación superior. El muestreo fue no probabilístico por conveniencia trabajando así con 31 estudiantes del curso de VI semestre Estructuras y Psicopatología del programa de psicología de la Corporación Universitaria Minuto de Dios - Centro Regional Ibagué. El enfoque metodológico es cuantitativo de diseño cuasiexperimental de tipo transeccional con aplicación pre y post del instrumento Lawson's Classroom Test of Scientific Reasoning. Entre la 
aplicación pre y post de dicho test se instruyó a los estudiantes para que utilizaran la estrategia ABP en el desarrollo de una actividad del curso mencionado. Dentro de los resultados obtenidos se encuentra, en cuanto a la clasificación del test en los niveles concreto, formal y postformal un leve aumento de la cantidad de sujetos que pasaron de la clasificación concreto a la formal. Sin embargo, con el principal análisis de dichos resultados se pudo concluir que las diferencias entre fase y fase no son significativas respecto a lo esperado, debido a factores como la limitación de tiempo para la aplicación del ABP.

PALABRAS CLAVES: Razonamiento, aprendizaje basado en problemas, educación.

ABSTRACT: The reasoning ability allows the search of information, the analysis of the same and give solution to a certain problem situation to solve; For its strengthening, it has become necessary to include didactic strategies in the teaching-learning processes that facilitate their development, one of these strategies is problem-based learning. The present research aimed to determine the influence of the Problem Based Learning strategy on the level of reasoning of a group of higher education students. Sampling was non-probabilistic for convenience working with 31 students from the course of VI semester Structures and Psychopathology of the psychology program of the University Corporation Minuto de Dios Ibagué Regional Center. The methodological approach is quantitative of quasi-experimental design of transectional type with pre and post application of the Lawson s Classroom Test of Scientific Reasoning. Between the pre and post application of this test students were instructed to use the ABP strategy in the development of an activity of the mentioned course. Within the results obtained, the classification of the test in the concrete, formal and postformal levels is a slight increase in the number of subjects that went from the concrete to the formal classification.
However, with the main analysis of these results it was possible to conclude that the differences between phase and phase are not significant in relation to the expected, due to factors such as the time limitation for the application of the ABP.

KEYWORDS: Reasoning, problem-based learning, education.

En el marco de los procesos de enseñanza aprendizaje se han hecho relevantes acciones para el desarrollo de habilidades por parte de los estudiantes para garantizar la aplicabilidad de lo aprendido a nivel teórico no solo en el período de formación académica, sino también en el contexto correspondiente a su desempeño profesional.

Este artículo tiene como propósito compartir los resultados encontrados al llevar a cabo la aplicación de un test de razonamiento científico Lawson's Classroom Test of Scientific Reasoning (Lawson, 1978), de manera previa y posterior a la implementación de la estrategia ABP para el desarrollo de una actividad académica de un curso del programa de psicología de una institución de educación superior en Colombia.

La demanda y necesidad de profesionales con habilidades que les permitan evidenciar además del saber teórico o del conocer, el saber aplicar los conocimientos in situ, ha hecho que desde el contexto educativo se replanteen constantemente las estrategias utilizadas no solo para enseñar conceptos sino para avanzar al desarrollo de habilidades de pensamiento, éstas, consideradas como "las herramientas de que dispone el alumno para procesar los contenidos y profundizar en el conocimiento" (Lara, 2012, p.90).

Para el desarrollo de este estudio se tuvo en cuenta la habilidad razonamiento científico, ésta permite al estudiante hacer un ejercicio constante de aplicación que lo lleva a converger los aspectos teóricos con los retos que implica la 
práctica en cuanto a la búsqueda de información, el análisis, el establecimiento de hipótesis, la toma de decisiones y la solución de determinada situación que representa un problema. "A medida que el adolescente aprende a utilizar la lógica proposicional, empieza a abordar los problemas de un modo más sistemático. Formula hipótesis, determina cómo compararlas con los hechos y excluye las que resultan falsas" (Linares, sf, p.18).

Lo anteriormente mencionado lleva a que se considere de importancia evidenciar los resultados obtenidos al aplicar estrategias como el aprendizaje basado en problemas, para a partir de dichos resultados aportar información que contribuya a la justificación de la necesidad de fortalecer la planeación curricular en cuanto a la inclusión de estrategias didácticas como el ABP y otras para el planteamiento de aspectos metodológicos que permitan desarrollar mejores habilidades en la formación profesional de los estudiantes.

La Corporación Universitaria Minuto de Dios UNIMINUTO Centro Regional Ibagué, es una institución de educación superior que inició sus actividades en la ciudad de lbagué en el año 2011 y desde entonces se ha ocupado de la mejora de sus procesos académicos en miras a formar profesionales altamente calificados y con sentido de responsabilidad social.

Uno de los programas profesionales allí ofertados es el de Psicología, donde hacía el sexto semestre de formación académica se ofrece el curso de Estructuras y Psicopatología, en este curso los estudiantes comienzan su acercamiento al tema relacionado con el comportamiento disfuncional o "anormal" del ser humano; a través de la revisión de lecturas y videos van conociendo las diferentes patologías mentales y su respectiva clasificación, además, a partir de dichos conocimientos los estudiantes vislumbran la importancia de su rol profesional en la sociedad especialmente en contextos relacionados con la salud mental. Para el desarrollo de la investigación se tomó como escenario el curso Estructuras y Psicopatología, se planteó de manera particular para el desarrollo de una de sus temáticas una situación problema en donde se incluyeron los elementos ya mencionados (trastornos mentales y el rol propositivo del estudiante frente a los mismos), y a partir de dicha situación problema los estudiantes debían aplicar la estrategia ABP con el fin de dar respuesta a lo propuesto.

Ahora bien, tomando como referencia algunos de los estudios previamente realizados por otros autores y que guardan relación con lo expuesto en el presente artículo especialmente en lo referente al uso de estrategias, particularmente $A B P$, se hace mención a la investigación de Gómez, F., Rivas, I., Mercado, F., y Barjola, P. (2009), la cual muestra principalmente los resultados de la aplicación de la estrategia ABP con la intención de contribuir al desarrollo de competencias profesionales. En esta investigación los resultados relevantes arrojaron datos como que el $90 \%$ de los estudiantes consideran que el ABP ofrece mejores ventajas de aprendizaje en comparación con un método tradicional; lo que indica que el uso de estrategias didácticas de este tipo contribuye al fortalecimiento y/o desarrollo de competencias en los estudiantes.

En la investigación basada en el enfoque de aprendizaje constructivista y retomando de éste dos estrategias: el aprendizaje colaborativo y el aprendizaje basado en problemas; Armenta, Salinas \& Mortera-Gutiérrez, (2013), plantearon en sus conclusiones "que aplicar la técnica ABP en una actividad académica impartida en línea, propicia las interacciones necesarias para el aprendizaje colaborativo", facilitando igualmente el desarrollo de otras habilidades tales como: el razonamiento crítico, aprendizaje auto dirigido, investigación a nivel individual, análisis y resolución de problemas. 
Por otra parte, Pacheco, García, y García (2012), en la investigación denominada ABP: llustración de su aplicación en psicología de la instrucción, muestran en sus resultados que el uso de este tipo de estrategias mejoró de manera considerable las habilidades de los estudiantes para realizar un trabajo colaborativo, así como también, evidenció la necesidad de promover el uso de este tipo de estrategias, dado a que en muchas ocasiones las limitaciones para investigar en este ámbito se relacionan con la resistencia de algunos docentes frente al uso de las mismas, prefiriendo la tradicional trasmisión de conocimientos como herramienta de enseñanza.

De igual manera, Ley, M. (2014), centra su investigación en determinar la efectividad del aprendizaje basado en la resolución de problemas en el desarrollo de la metacognición de estudiantes universitarios de una universidad virtual mexicana. En el estudio se encontró que los estudiantes que participaron en la investigación, poseen conocimiento de algunas estrategias metacognitivas dentro de ellas el ABP; no obstante, ese conocimiento no se transforma en acciones concretas que inciden en un mejor rendimiento académico.

Por otra parte, la OCDE ofrece el Panorama de las habilidades 2013. Primeros resultados de la encuesta sobre las habilidades de los adultos, en donde se evidencia la necesidad de fortalecer las competencias en la educación especialmente en las áreas de lenguaje y cálculo numérico; lo que apoya la justificación para desarrollar investigaciones como la descrita en el presente artículo con el fin de mostrar la influencia significativa que conlleva la aplicación de estrategias didácticas como el ABP para el desarrollo de habilidades de razonamiento en los estudiantes de educación superior.

\section{MARCO TEÓRICO}

Para una mejor comprensión de lo desarrollado, se abordan a continuación los principales fundamentos conceptuales y teóricos sobre los que se enmarca el propósito de la investigación en mención. El primer concepto relevante es el de razonamiento, para abordar el tema de razonamiento como habilidad cognitiva, es necesario realizar una revisión previa centrada en la teoría propuesta por Jean Piaget, quien planteó 4 etapas que comprenden el desarrollo cognitivo, estas etapas son: Sensorio-Motora, pre- operacional, operaciones concretas y operaciones formales (Pozo y Gómez, 2006); haciendo énfasis en la etapa de las operaciones formales en la que se esboza el uso de una habilidad más compleja a nivel de pensamiento, denominada razonamiento científico; de ésta última se cita la importancia de su enseñanza no solo a partir de los primeros niveles de escolarización sino también en el nivel de educación superior.

La etapa de las operaciones formales es la cuarta y última descrita por Piaget en su teoría del desarrollo del pensamiento, en esta se plantea que el individuo alcanza la capacidad de resolver situaciones de forma lógica, lo que conlleva a que su pensamiento se vuelva cada vez más científico debido a la necesidad de analizar elementos tanto palpables como no palpables para establecer hipótesis frente a la solución de situaciones específicas. De igual forma, el individuo pasa de pensar en la realidad concreta y tangible a experimentar la visualización de lo que podría ser, es decir, lo intangible, cuestión que implica la habilidad para realizar abstracciones y está ligada a dos aspectos primordiales como son la práctica y la experiencia (Pozo y Gómez, 2006).

El pensamiento del individuo en la etapa de las operaciones formales empieza a desarrollar una característica de tipo científico, esto quiere decir, que va adquiriendo un pensamiento mucho más 
complejo que le permite trabajar bajo supuestos los cuales de alguna manera se basan en lo que ya se conoce como concreto, solo que para este punto ya va desarrollando la capacidad de ir más allá de lo que percibe a simple vista, lo que se traduce en la posibilidad de razonar de manera hipotética-deductiva; dicho en otras palabras, "Al superar su realidad inmediata, las operaciones formales permiten, no sólo buscar explicaciones de los hechos que vayan más allá de la realidad aparente, sino además someterlas a comprobaciones sistemáticas" (Pozo y Gómez, 2006, p. 78).

Así pues, se habla entonces del razonamiento como la habilidad cognitiva que se fortalece a través del ejercicio del estudiante de plantearse preguntas, observar, generar hipótesis y comprobarlas (Lawson, 2008). En concordancia con lo anterior, se evidencia la necesidad de desarrollar el pensamiento formal para llegar a un razonamiento de tipo científico que enriquezca otras habilidades a nivel cognoscitivo en el individuo, así, este podrá desenvolverse en situaciones no solo de índole académico sino también de carácter profesional y cotidiano.

Ahora bien, para conocer el nivel de razonamiento de los estudiantes es necesario saber que éste se ha clasificado en seis habilidades y tres niveles, los cuales son medidos a través del Test de razonamiento científico en el salón de clases de Lawson. Las habilidades son: Conservación de magnitudes físicas o masa, pensamiento de proporcionalidad, identificación y control de variables avanzada, pensamiento probabilístico, pensamiento combinatorio; $y$, pensamiento correlacional. En cuanto a la clasificación del razonamiento en niveles se estipulan tres: concreto, formal y postformal.

Otro concepto relevante, es el relacionado con la definición de la estrategia didáctica de aprendizaje basado en problemas, pero entiéndase inicialmente que una estrategia didáctica como tal es aquella que "se involucra con la selección de actividades y prácticas pedagógicas en diferentes momentos formativos, métodos y recursos en los procesos de Enseñanza-Aprendizaje" (Velasco y Mosquera, 2010, p.1-2). Las estrategias son entendidas como una forma de planeación que llevan al cumplimiento de propósitos dentro del desarrollo curricular para el proceso de enseñanza-aprendizaje.

De igual forma, cabe mencionar también que la aplicación de estrategias didácticas debe enmarcarse en un modelo de aprendizaje, para este caso se considera el modelo constructivista, "El paradigma constructivista propone secuencias de enseñanza, esto significa poner al alumno ante experiencias donde él construya sus conocimientos desde la memoria comprensiva, a través de situaciones en las cuales encuentre un equilibrio adecuado entre la lógica del saber y la lógica de su propia estructura y desarrollo cognitivo". (Espinoza, Sánchez, (2014), p.112).

Así pues, una de las estrategias didácticas que siguen el modelo constructivista es el aprendizaje basado en problemas (ABP). EI ABP tiene su sustento teórico en el constructivismo, que como su mismo nombre lo indica se centra en la manera en cómo se da la construcción del conocimiento (Armenta, Salinas, Mortera, 2013). Dicha construcción del conocimiento surge de la interacción del individuo (en este caso el estudiante) con su entorno y es allí donde elaboran los significados individuales frente a la realidad (Remy, 2004).

Esta estrategia didáctica es utilizada en el proceso de enseñanza-aprendizaje por parte de docentes de diversos niveles de educación, el principal propósito de ésta es el desarrollo de habilidades relativas al razonamiento, que como ya se ha visto incluye la capacidad de plantear hipótesis, analizar la información, realizar abstracciones, hacer búsqueda de información y tomar decisiones para la resolución del 
problema o situación propuesta como parte de la aplicación de dicha estrategia (ITESM, 2004).

Para el desarrollo de la estrategia didáctica se siguen una serie de pasos para la solución del problema establecido en relación con los contenidos de aprendizaje. Los pasos son ocho y se visualizan en la figura 1 .

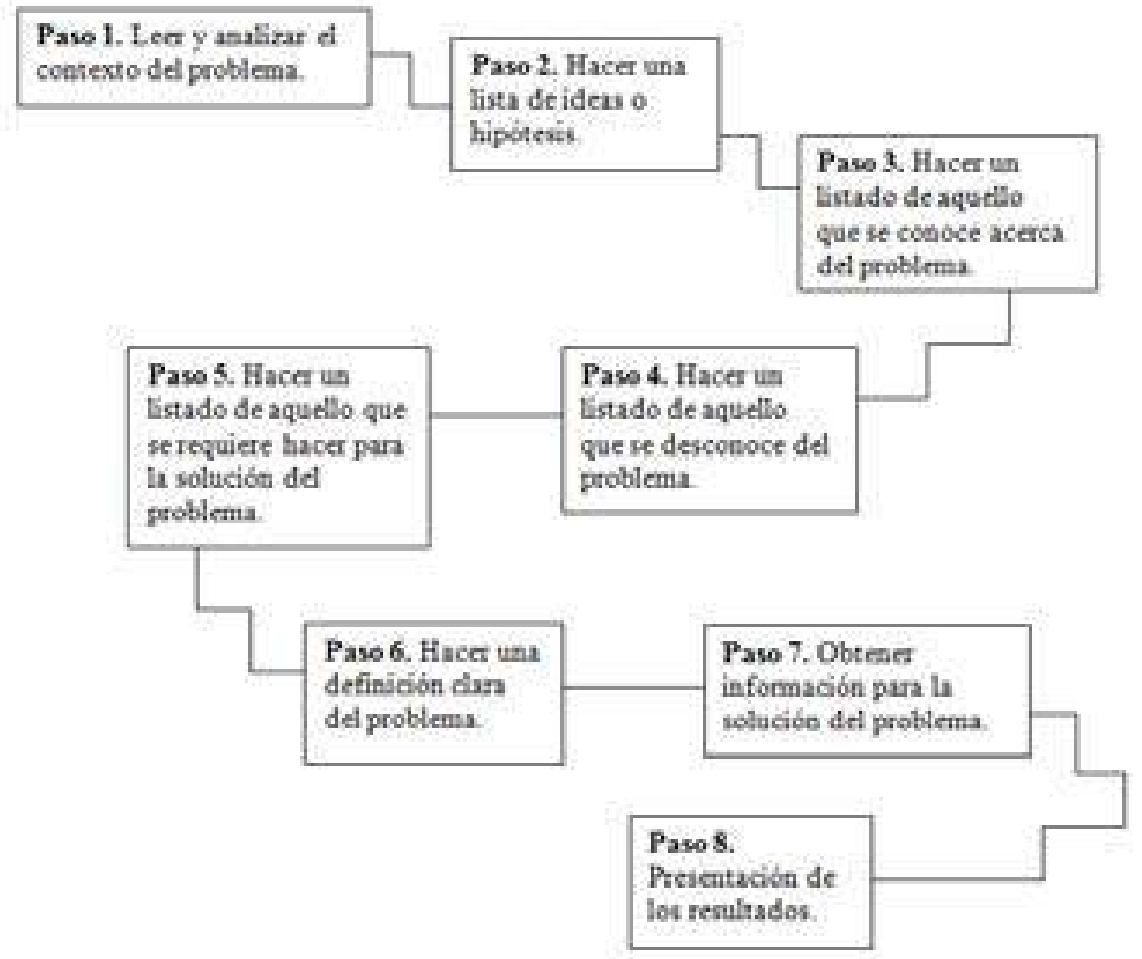

Figura 1. Pasos de la estrategia ABP. (SERVICIO, D. I. E. 2008).

\section{PROCEDIMIENTO METODOLÓGICO}

Se abordó la investigación a partir del paradigma positivista, el cual se "caracteriza por una definición clara de variables por estudiar, cada una de las cuales representa un constructo" (Valenzuela y Flores, 2013, p.604). El enfoque metodológico utilizado fue el cuantitativo, que se distingue por "la recolección de datos para probar hipótesis, con base en la medición numérica y el análisis estadístico" (Hernández, Fernández y Baptista, 2010, p.4). El diseño es cuasiexperimental con aplicación pre y post y de tipo transeccional o transversal, este diseño como plantea Hernández et al., (2010) permite la manipulación de una variable independiente para establecer su efecto sobre una o más variables dependientes; y la recolección de los datos se da en un tiempo y espacio único.

En relación con los participantes, la unidad de análisis fueron estudiantes de educación superior. La población corresponde aproximadamente a 110 estudiantes de sexto semestre de psicología de la Corporación Universitaria Minuto de Dios Centro Regional Ibagué que asisten al curso Estructuras y Psicopatología. Para la determinación de la muestra se realizó un muestreo no probabilístico por conveniencia debido a que se seleccionaron 31 estudiantes matriculados en dicho curso.

Para medir el razonamiento previo y posterior a la estrategia ABP se eligió el Lawson's Classroom 
Test of Scientific Reasoning (Lawson, 1978) que es un cuestionario cuyo objetivo es medir las seis habilidades de razonamiento relacionadas con el aprendizaje de las ciencias, y los tres niveles de razonamiento. El test está formado por 24 reactivos de opción múltiple, donde la evaluación se hace por pares de preguntas ya que un reactivo corresponde a la respuesta y el otro a la justificación de dicha respuesta.

La aplicación previa del instrumento se realizó a los 31 estudiantes de la muestra en el curso ya mencionado, posterior a la aplicación se explicó y entregó a los estudiantes un documento en el que evidenciaron en qué consiste la estrategia didáctica aprendizaje basado en problemas y los pasos para su realización; así como también se les asignó la situación o escenario el cual planteaba el reto a cada equipo de desarrollar estrategias de divulgación sobre un trastorno mental (uno por equipo), para esto debían aplicar cada uno de los pasos de la estrategia ABP.

Los estudiantes conformaron equipos de seis personas aproximadamente y tuvieron dos semanas para desarrollar la actividad completa y hacer la respectiva entrega. En el transcurso de las dos semanas de realización de la actividad recibieron asesoría por parte de la docente para verificar cómo iba el cumplimiento de los pasos y asignar las especificaciones para la entrega

Tabla 1 del reporte escrito. Luego de la entrega de los reportes escritos, se procedió a la aplicación del test de razonamiento en su fase post.

Para la exploración de los datos con estadística descriptiva, se introdujeron las respuestas del test de Lawson en el software Statistical Package for the Social Sciences (SPSS), un programa que "facilita crear un archivo de datos en una forma estructurada y también organizar una base de datos que puede ser analizada con diversas técnicas estadísticas". (Castañeda, Cabrera, Navarro y Vries, 2010, p.15).

Adicional a lo anterior, también se revisaron supuestos de normalidad y homogeneidad de varianzas para las comparaciones de las medias entre grupos en pruebas paramétricas tal como sugiere Sheskin (2007). Para la revisión de la relación entre variables se aplicó la prueba de rangos signados de Wilcoxon debido a que los datos no resultaron normales.

\section{ANÁLISIS Y DISCUSIÓN DE RESULTADOS}

A continuación, se comparten los resultados más relevantes obtenidos a partir de la realización del trabajo de campo. Como se mencionó en el apartado anterior respecto al instrumento, su calificación de respuestas acertadas se realiza a partir del número de pares de respuestas contestados de manera correcta. En la tabla 1 se evidencian los pares acertados tanto en la fase de aplicación pre como en la post.

Resultados estadísticos

\begin{tabular}{|l|c|c|}
\hline \multicolumn{1}{|c|}{ Estadísticos } & Total de pares pretest & Total de pares postest \\
\hline Media & 1.97 & 1.87 \\
\hline Mediana & 2.00 & 1.00 \\
\hline Moda & 1 & 1 \\
\hline Desviación Típica & 1.329 & 1.544 \\
\hline Varianza & 1.766 & 2.383 \\
\hline Mínimo & 0 & 0 \\
\hline Máximo & 5 & 6 \\
\hline
\end{tabular}

Fuente: elaboración a partir de los resultados a través del SPSS. 
El promedio de pares correctos fue de 5 en el pretest y de 6 en el postest. Esto indica un leve, aunque no significativo aumento en los aciertos de respuesta esperados para la prueba.

Ahora bien, como se ha mencionado en el sustento teórico y como propósitos de la investigación, se muestra en la tabla 2 el paralelo entre los resultados pre y post a la aplicación del test en relación con los indicadores correspondientes al nivel de razonamiento.

Tabla 2

Nivel de razonamiento del pretest y Postest

\begin{tabular}{|l|c|c|c|c|}
\hline & \multicolumn{2}{|c|}{ Pretest } & \multicolumn{2}{c|}{ Postest } \\
\hline & $N^{\circ}$ & $\%$ & $N^{\circ}$ & $\%$ \\
\hline Concreto & 28 & 90.3 & 26 & 83.9 \\
\hline Formal & 3 & 9.7 & 5 & 16.1 \\
\hline Total & 31 & 100.0 & 31 & 100.0 \\
\hline
\end{tabular}

Fuente: elaboración a partir de los resultados a través del SPSS.

Esta segunda tabla ilustra que en la fase de aplicación pre, el $90.3 \%$ de los estudiantes están en un nivel de razonamiento concreto y el $9.7 \%$ en un nivel de razonamiento formal; mientras que en la fase de aplicación post, el 83.9\% de los estudiantes están en un nivel de razonamiento concreto y el $16.1 \%$ en un nivel de razonamiento formal. Lo anterior indica que

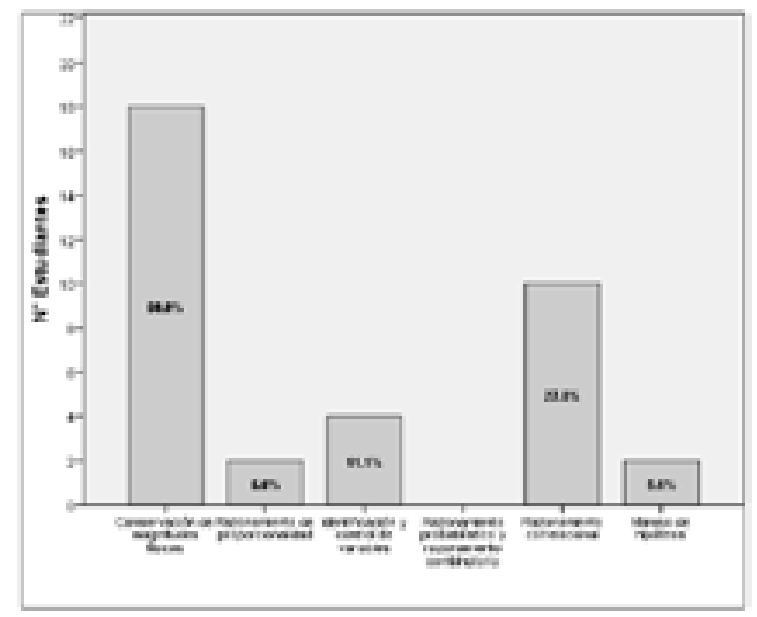

Figura 2. Habilidades de razonamiento pretest. postest. posterior a la aplicación de la estrategia ABP se evidencia un leve aumento de personas clasificadas en el nivel de razonamiento formal.

En cuanto a los resultados del test respecto a los indicadores correspondientes a las habilidades de razonamiento medidas por el mismo, en la figura 2 y 3 se pueden observar los resultados de la aplicación pre y post respectivamente.

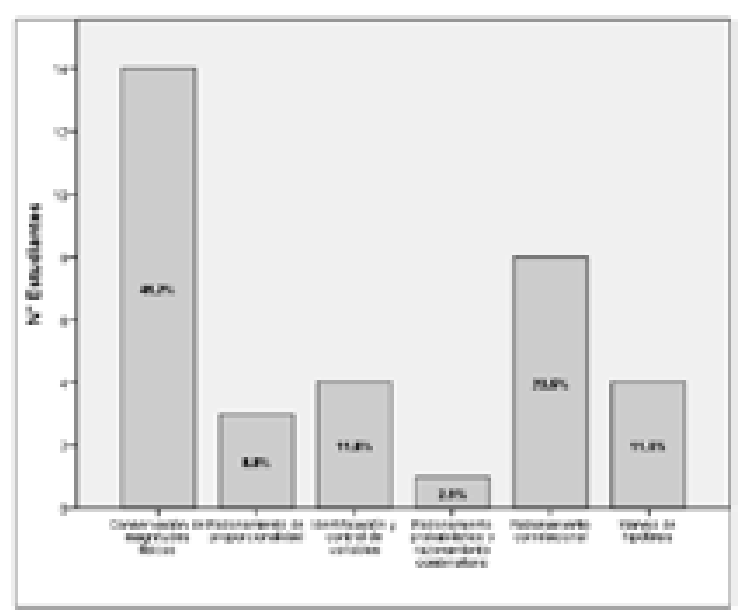

Figura 3. Habilidades de razonamiento

Fuente: elaboración a partir de los resultados a través del SPSS. 
De las dos figuras vistas se analiza un leve incremento entre la fase de aplicación pre y post, en los porcentajes de las habilidades de razonamiento proporcional de $5.6 \%$ aumenta a $8.8 \%$; razonamiento probabilístico y combinatorio de $0 \%$ aumenta a $2.9 \%$, y finalmente, en cuanto a la habilidad manejo de hipótesis de $5.6 \%$ aumenta a $11.8 \%$. Dichos incrementos muestran que luego de la aplicación de la estrategia ABP se logra una mejora en las habilidades ya mencionadas.

Los resultados obtenidos y analizados muestran que no hay diferencias significativas entre los resultados de la aplicación del Test de Lawson previo a la incursión de la estrategia didáctica ABP respecto a los resultados de su aplicación posterior.

Lawson (1978) propone en su instrumento de medición de razonamiento científico una clasificación de acuerdo al número de aciertos que se obtengan al responder su prueba (Lawson 's Classroom Test of ScientificReasoning); dicha clasificación establece tres niveles: concreto, formal y postformal. Para esta investigación los resultados han mostrado que en su aplicación pretest el $90.3 \%$ de los estudiantes presentaron un nivel de razonamiento concreto, mientras que el $9.7 \%$ presentaron un nivel de razonamiento formal.

Si bien los resultados del postest mostraron variaciones leves en los resultados, $83.9 \%$ de estudiantes para el nivel concreto y $16.1 \%$ para el nivel formal, dichas variaciones no son significativas respecto a lo esperado, aspecto que se corroboró con la aplicación de la prueba Wilcoxon para muestras relacionadas, donde se confirma que no existen diferencias significativas entre la fase pre y post; esto coincide con la literatura donde se plantea que el razonamiento es una habilidad compleja de adquirir, estableciendo que por lo general las personas se limitan a las operaciones concretas dada su particularidad de tangible, asociado a lo accesible (Pozo y Gómez, 2006).

En términos generales, los sujetos de la muestra mantienen luego del desarrollo de la actividad haciendo uso de la estrategia $A B P$, su nivel de razonamiento concreto, dando cuenta así de que si bien el $A B P$ influye en el desarrollo de habilidades como la capacidad de plantear hipótesis, analizar la información, realizar abstracciones, hacer búsqueda de información y tomar decisiones para la resolución de un problema (ITESM, 2004), su aplicación en la actividad no evidenció el fortalecimiento de dichas habilidades asociadas al razonamiento, por lo que sería pertinente la incursión de este tipo de estrategias en la planeación curricular para garantizar que su aplicación constante fortalezca las habilidades ya mencionadas.

Finalmente, si bien las diferencias no son significativas, si se puede inferir a partir de la observación de resultados, que de cinco aciertos en la fase pretest se pasa a seis aciertos en la fase postest, lo que si bien no es representativo, si permite hipotetizar sobre la posibilidad de aumentar el número de aciertos en la medida en que se dé una mayor aplicación de estrategias de aprendizaje como la ya mencionada; esto en concordancia con los resultados de la OCDE (2013) en su Panorama de las habilidades, donde evidencian la necesidad de fortalecer las competencias en la educación de los adultos especialmente en las áreas de lenguaje y cálculo numérico.

\section{CONCLUSIONES}

Con base en los resultados obtenidos en la investigación que buscaba determinar la influencia de la estrategia ABP sobre el nivel de razonamiento de estudiantes de educación superior a través de la identificación y el análisis del nivel de su nivel de razonamiento previo y posterior a la aplicación del ABP, se concluye que según Lawson (1978) quien en 
su instrumento de medición de razonamiento científico establece una clasificación de tres niveles: concreto, formal y postformal; en los sujetos de la muestra se evidencia un ligero aumento en la clasificación formal; lo que de alguna manera demuestra que existe influencia de la estrategia $A B P$ en el nivel de razonamiento de los estudiantes.

En concordancia con lo anterior, los resultados demuestran que la mayoría de los estudiantes de la muestra fueron clasificados en el nivel de razonamiento concreto, lo cual corrobora el planteamiento de Pozo y Gómez (2006) acerca de que por lo general las personas se limitan a las operaciones concretas dada su particularidad de tangible, asociado a lo accesible.

Al consolidar los resultados y realizar la discusión de los mismos en contraste con las propuestas teóricas tomadas como referencia para la investigación, se puede analizar a partir de los datos obtenidos que el razonamiento es una habilidad cognitiva que se fortalece a través del ejercicio del estudiante de plantearse preguntas, observar, generar hipótesis y comprobarlas (Lawson, 2008).

En lo referente a los alcances y limitaciones, se concluye que uno de los aspectos a tener en cuenta en lo concerniente a limitaciones es el tiempo de aplicación del instrumento y uso de la estrategia ABP, ya que el abordaje en dos semanas se considera un tiempo insuficiente para lograr cambios significativos en los resultados de la fase post del test de Lawson; por lo que se sugiere el desarrollo de actividades adicionales utilizando la estrategia para consolidar la aprehensión y efectividad en el uso de los pasos, lo que de manera directa fortalece la habilidad de razonamiento como ya se ha expuesto en la teoría que soporta el estudio.

Es importante indicar que el desarrollo de este tipo de investigaciones aporta conocimiento al ámbito de la educación dado a que su enfoque y sus resultados muestran la importancia de incrementar el uso de estrategias didácticas del tipo ya descrito, para así fortalecer las habilidades de los estudiantes y consolidar su proceso de aprendizaje en miras no solamente a que el estudiante obtenga unos conceptos y procedimientos, sino que sea capaz de desarrollar destrezas para su aplicación en contextos reales a través de la realización de análisis críticos, rastreo de información, formulación de soluciones y aplicación de las mismas para dar respuesta a las demandas contemporáneas del contexto local y mundial.

Otro aspecto relevante es el alcance de la investigación, si bien se habla de estudiantes de educación superior, la muestra corresponde a un grupo de treinta y un estudiantes, sugiriéndose entonces ampliar el número de sujetos de dicha muestra con el fin de obtener un mayor número de datos para establecer las comparaciones en las aplicaciones pre y post del instrumento.

A partir de lo realizado cabe mencionar la importancia de establecer otras investigaciones relacionadas con el tema de la presente, conforme a ello, se proponen como posibles preguntas de investigación, ¿Qué factores cognitivos influyen en la habilidad de razonamiento científico de los estudiantes de educación superior?, ¿Cuál estrategia de aprendizaje puede desarrollar con mayor eficacia el razonamiento científico en estudiantes de educación superior?

Finalmente, se considera relevante indagar por un criterio referente a lo que los estudiantes opinan acerca del uso de la estrategia ABP, qué aprendizajes consideran obtienen con el uso de ésta, qué hallazgos realizan de su proceso de aprendizaje y las prácticas que llevan a cabo para el desarrollo de actividades extraclase; ya que estos aspectos pueden enriquecer el soporte para el uso de estrategias didácticas de aprendizaje como el ABP para incrementar el nivel de razonamiento en los estudiantes. 


\section{REFERENCIAS}

Armenta, M. D., Salinas, V., Mortera-Gutiérrez, F. (2013). Aplicación de la técnica educativa aprendizaje basado en problemas para capacitación a distancia (E-learning). Revista Iberoamericana de Educación a Distancia, 16 (1), 57-83. Recuperado de http://catedra.ruv.itesm. $\mathrm{mx} /$ handle/987654321/661

Castañeda, M. B. (2010). Procesamiento de datos y análisis estadísticos utilizando SPSS: Un libro práctico para investigadores $y$ administradores educativos. EDIPUCRS. Recuperado de https://books.google.com.co/ books?hl=es\&lr=\&id=XsxqTVs9-2QC\&oi $=$ fnd $\&$ pg $=P A 8 \&$ ots $=8 M 36$ il JWsa $\&$ sig $=N \mathrm{~J}$ 9oR_da_aOMnOpLESUZZxKTYjc\&redir_ esc $=y \# v=$ onepage $\& q \&=$ false

Coral, A. L. (2013). Desarrollo de habilidades de pensamiento y creatividad como potenciadores de aprendizaje. Revista Unimar, 30(1).

Espinoza Melo, C. C., \& Sánchez Soto, I. R. (2014). Aprendizaje basado en problemas para enseñar y aprender estadística y probabilidad.

Fuentes, M. G. L. (2014). El Aprendizaje Basado en la Resolución de Problemas y su efectividad en el Desarrollo de la Metacognición. Educatio siglo XXI, 32(3 Noviembr), 211-230.

Gómez, F., Rivas, I., Mercado, F., Barjola, P. (2009). Aplicación interdisciplinar del aprendizaje basado en problemas (ABP) en ciencias de la salud: una herramienta útil para el desarrollo de competencias profesionales. Red U. Revista de Docencia Universitaria, 4, 1-19. Recuperado de http://redu.net/ redu/index.php/REDU/article/view/108
Hernández, R., Fernandez, C. Baptista, P. (2010). Metodología de la investigación. México D.F, México: McGraw-Hill Interamericana.

Instituto Tecnológico y de Estudios Superiores de Monterrey (México). (2004). El aprendizaje basado en problemas como técnica didáctica. Universitat de Barcelona. Departament de Dret Mercantil, Dret del Treball i de la Seguretat Social. Recuperado de http:// sitios.itesm.mx/va/dide/documentos/infdoc/abp.pdf

Lawson, A. (2009).Basic inferences of scientific reasoning, argumentation, and discovery.ScienceEducation,336-364. doi: 10.1002/sce.20357

Linares, A. R. (s.f). Desarrollo cognitivo: Las teorías de Piagety Vygotsky. Recuperado de http://www.paidopsiquiatria.cat/files/ teorias_desarrollo_cognitivo_0.pdf

OCDE (2013). Panorama de las habilidades 2013. Primeros resultados de la encuesta sobre las habilidades de los adultos.París, Francia. Recuperado de http://www.redetis.iipe.unesco. org/publicaciones/panorama-de-lashabilidades-2013- primeros-resultadosde-la-encuesta-sobre-las-habilidadesde-los-adultos-ocde/\#.VQh3- NKG9fA

Pozo, J.I. Gómez C, M.A. (2006). Aprender $y$ enseñar ciencia. Del conocimiento cotidiano al conocimiento científic. Madrid, España: Ediciones Morata.

Remy, H. D. Z. (2004). El constructivismo en los procesos de ensenanza-aprendizaje en el siglo XXI. Plaza y Valdes. 
Sanz, D. P., Sánchez, J. N. G., \& Martín, J. G. (2012). ABP: ilustración de su aplicación en psicología de la instrucción. International Journal of Developmental and Educational Psychology, 2(1), 3139.

SERVICIO, D. I. E. (2008). Aprendizaje Basado en Problemas. Guías rápidas sobre nuevas metodologías. Recuperado de http://innovacioneducativa.upm. es/guias/Aprendizaje_basado_en_ problemas.pdf

Valenzuela, J. Flores, M. (2012). Fundamentos de investigación educativa (Vol. 2: El proceso de investigación educativa). Monterrey, México: Editorial Digital del Tecnológico de Monterrey.

Velasco, M. Mosquera. (2010). Estrategias Didácticas para el Aprendizaje Colaborativo.PAIEP. Consultado el 12 de abril de 2013, en:http://acreditacion. udistrital.edu.co/flexibilidad/estrategias_ didacticas_aprendizaje_colaborativo.pdf 